\title{
GRAVITATIONAL WAVES IN GENERALISED BRANS-DICKE THEORY
}

\author{
B.K.Sahoo ${ }^{1}$ and L.P.Singh ${ }^{2}$ \\ Department of Physics, \\ Utkal University,Bhubaneswar-751004,India \\ 1 bijaya@iopb.res.in \\ 2 lambodar@iopb.res.in
}

\begin{abstract}
We have solved Cosmological Gravitational Wave(GW) equation in the frame work of Generalised Brans-Dicke(GBD) theory for all epochs of the Universe.The solutions are expresed in terms of the present value of the Brans-Dicke coupling parameter $\omega(\phi)$.It is seen that the solutions represent travelling growing modes for negative values of $\omega_{0}$ for all epochs of the Universe.
\end{abstract}

Key words:Gravitational waves,growing modes.

PACS NO:98.80.-K,98.80.cq

\section{INTRODUCTION}

General Relativity (GR) predicts that perturbations of a background spacetime generate gravity waves. The general results concerning gravitational waves have been investigated by numerous authors long ago[1].In the context of GR cosmologies,Grishchuk [2] has shown that the varying gravitational field of the expanding Universe would amplify zero -point fluctuations, and lead to the formation of relic gravitons .Gravitons interact very weakly with matter and other fields, and only at energies of order of the planck enegy $\left(10^{19} \mathrm{GeV}\right)$ will the gravitational interaction become as strong as the electromagnetetic interaction.Therefore, the relic gravitons must have decoupled from the dynamics of the Universe at extremely early times,and offer a unique way to probe the structure and evolution of the early Universe.Even without detecting them,we can still get useful information by studying the consequences of their presence in several processes of the cosmic history ;for example,primordial nucleosynthesis and the anisotropy they would induce in the microwave background . Thus, the idea of using gravitational radiation as probe of the early Universe has turned into a realistic possibility. The subject of gravitational radiation within Brans-Dicke (BD) theory was carried out by Wagoner[3]. This was concerned with gravitational waves generated in the weak field limit.Barrow[4] has derived the cosmological gravitational wave equation in some more general scalar tensor theories of gravity and solved the wave equation for universe in BD theory where the coupling function is a constant.However,in the generalised Brans-Dicke(GBD) theory of gravity where the coupling function $\omega=\omega(\phi)$, the gravitational wave equation is solved by Barrow for only vacuum and radiation dominated epochs of the Universe for a particular choice of the coupling function $\omega(\phi)$. Thus the work seems to be incomplete in GBD theory of gravity.Here we investigate the cosmological solutions of the gravitational wave equation for all epochs of the Universe in GBD theory by allowing the coupling parameter to run with scalar field $\phi$ following the approach adopted in our recent work[5]. The solutions are expresed in terms of present value of the coupling parameter $\omega(\phi)$ i.e,in terms of $\omega_{0}$. An outline of this paper is as follows.In the section II we have derived the solutions. The solutions are expresed in terms of $\omega_{0}$ in section III for all epochs of the Universe and shown that for negative values of $\omega_{0}$ solutions represent growing modes..We conclude with section IV. 


\section{WAVE EQUATION}

The gravitational field equations are given below $[6]$

$$
\begin{aligned}
& R_{\mu \nu}=\frac{8 \pi G}{\phi}\left[T_{\mu \nu}-\frac{1+\omega}{2 \omega+3} g_{\mu \nu} T\right]+\frac{\omega}{\phi^{2}} \phi,{ }_{\mu} \phi,{ }_{\nu}+\frac{1}{\phi} \phi, \mu \nu-\frac{1}{2} \frac{g_{\mu \nu}}{\phi} \frac{d \omega / d \phi}{2 \omega+3} \phi_{, \sigma} \phi^{, \sigma}-g_{\mu \nu} \frac{1}{2 \omega+3}[\phi(d U / d \phi)+2(\omega+1) U] \\
& \square \phi=\frac{8 \pi}{2 \omega+3} T-\frac{d \omega / d \phi}{2 \omega+3} \phi_{, \sigma} \phi_{, \sigma}-\frac{2}{2 \omega+3}\left[\phi^{2}(d U / d \phi)-\phi U\right]
\end{aligned}
$$

When perturbation is applied to above equations, they become[4,7]

$$
\begin{aligned}
& \delta R_{\mu \nu}=\delta\left(\frac{8 \pi G}{\phi}\left[T_{\mu \nu}-\frac{1+\omega}{2 \omega+3} g_{\mu \nu} T\right]\right)+\delta\left(\frac{\omega}{\phi^{2}} \phi_{, \mu} \phi_{, \nu}\right)+\delta\left(\frac{1}{\phi} \phi_{, \mu \nu}\right)-\delta\left(\frac{1}{2} \frac{g_{\mu \nu}}{\phi} \frac{1}{2 \omega+3} \frac{d \omega}{d \phi} \phi_{, \sigma} \phi^{, \sigma}\right)-\delta\left(g _ { \mu \nu } \frac { 1 } { 2 \omega + 3 } \left[\phi \frac{d U}{d \phi}+\right.\right. \\
& 2(\omega+1) U]) \\
& \delta \square \phi=\delta\left(\frac{8 \pi T}{2 \omega+3}\right)-\delta\left(\frac{1}{2 \omega+3} \frac{d \omega}{d \phi} \phi_{, \sigma} \phi_{,}^{\sigma}\right)-\delta\left(\frac{2}{2 \omega+3}\left[\phi^{2} \frac{d U}{d \phi}-\phi U\right]\right)
\end{aligned}
$$

Where the perturbed metric is

$\tilde{g}_{\mu \nu}=g_{\mu \nu}+\delta g_{\mu \nu}$

$=g_{\mu \nu}+h_{\mu \nu}, \quad$ where, $\delta g_{\mu \nu}=h_{\mu \nu}$

leading to the Ricci tensor, $\tilde{R}_{00}=R_{00}+\delta R_{00}$.

The energy-momentum tensor is given by , $\tilde{T}^{00}=T^{00}+\delta T^{00}=T^{00}+\delta \rho$,

The trace of energy-momentum tensor is , $\tilde{T}=T+\delta T=T+\delta \rho-3 \delta P$.

For the d'Alembertian of the scalar field we find , $\delta \square \phi=\delta \ddot{\phi}+a \dot{a} h^{i j} \dot{\phi}-\frac{1}{2 a^{2}} h_{i j} \dot{\phi}+3 \frac{\dot{a}}{a} \delta \dot{\phi}-\frac{\nabla^{2}}{a^{2}} \delta \phi$.

We adopt the synchronous gauge, $h_{0 \mu}=0$

The time time component of perturbed Ricci tensor is[8], $\delta R_{00}=\frac{1}{a^{2}}\left[\ddot{h_{i j}}-2 \frac{\dot{a}}{a} h_{i j}+2\left(\frac{\dot{a}^{2}}{a^{2}}-\frac{\ddot{a}}{a}\right) h_{i j}\right]$. When $\phi=$ constant,all the perturbed equations will reduce to perturbed equation of Eienstein. The perturbations to the metric that represent weak gravitational waves can be expressed as $[9]$

with,

$$
h_{i j}(t, x)=\int d^{3} k h_{i j}^{(k)}(t, x),
$$

$$
h_{i j}^{(k)}(t, x)=\frac{1}{a^{2}(t)} Y_{k}(t) \zeta_{i j}(k, x),
$$

Where the functions $Y_{k}(t)$ and $\zeta_{i j}(k, x)$ satisfy

$$
\begin{gathered}
\left(\nabla^{2}+k^{2}\right) \quad \zeta_{i j}(k, x)=0, \\
\ddot{Y}_{k}+f(t) \dot{Y}_{k}+g(t) Y_{k}=0,
\end{gathered}
$$

where $\quad f(t)=\frac{\dot{\phi}}{\phi}-\frac{\dot{a}}{a}$,

$g(t)=\frac{k^{2}}{a^{2}}+4 \frac{\dot{a}^{2}}{a^{2}}-\frac{8 \pi}{\phi}\left[\frac{2(1+\omega)}{2 \omega+3} \rho-\frac{2 \omega}{2 \omega+3} P\right]-\frac{1}{2 \omega+3} \frac{d \omega}{d \phi} \frac{\dot{\phi}^{2}}{\phi}+\frac{1}{2 \omega+3}\left[\phi \frac{d U}{d \phi}+2(\omega+1) U\right]$

In the above equations, $\mathrm{a}(\mathrm{t})$ is the scale factor, $i, j=1,2,3 ; \nabla^{2}$ is the spatial Laplacian operator, an over dot indicates derivatives with respect to the synchronous cosmic time , t, and $k=|\vec{k}|=\frac{2 \pi a}{\lambda}$ with $\vec{k}$ being the comoving wave vector. The eigen functions $\zeta_{i j}(k, x)$ can be written in terms of plane -wave solutions $\exp ( \pm i \vec{k} \cdot \vec{x})$ times a constant polarization tensor and using the field equations in the zero curvature FRW model we can simplify (3) and recast it as [4]

$$
\ddot{Y}_{k}+\left[\frac{\dot{\phi}}{\phi}-\frac{\dot{a}}{a}\right] \dot{Y}_{k}+\left[\frac{k^{2}}{a^{2}}-2 \frac{\ddot{a}}{a}-2 \frac{\dot{a}}{a} \frac{\dot{\phi}}{\phi}-\frac{d \omega / d \phi}{2 \omega+3} \frac{\dot{\phi}^{2}}{\phi}+\frac{1}{2 \omega+3}\left(\phi \frac{d U}{d \phi}+2(\omega+1) U\right)\right] Y_{k}=0
$$

This is the Gravitational wave equation in more general scalar-tensor theory of gravity. 


\section{SOLTUIONS IN GBD THEORY}

The generalised Brans-Dicke scalar-tensor theory of gravity is characterised by the generalisation of the coupling function $\omega$ to a function of $\phi$; and $U=\frac{d U}{d \phi}=0$. We introduce the conformal time $\eta$ defined by $d t=a d \eta$

Each component $h_{i j}^{(k)}$ of the gravitational wave perturbations can be written as[4]

$$
h_{i j}^{(k)}(\eta, x)=\frac{1}{R(\eta)} \mu(k, \eta) \zeta_{i j}(k, x)
$$

Here $R(\eta)=a(\eta) \phi^{1 / 2}(\eta)$ and

$$
\mu^{\prime \prime}(k, \eta)+\left[k^{2}-\frac{R^{\prime \prime}}{R}-\frac{d \omega / d \phi}{2 \omega+3} \frac{\phi^{2}}{\phi}\right] \mu(k, \eta)=0
$$

with the prime indicating derivatives with respect to $\eta$.

When $\omega(\phi)=$ constant, eqn.(6) becomes, $\mu^{\prime \prime}(k, \eta)+\left[k^{2}-\frac{R^{\prime \prime}}{R}\right] \mu(k, \eta)=0$

which represents Gravitational wave equation in Brans-Dicke scalar-tensor theory of gravity.The solution given by eqn(6) is formally identical to the corresponding one obtained in the general relativistic case,but with the quantity $R(\eta)$ defined by eqn(5) replacing the scale factor $a(\eta)$.Equation (6) describes an oscillator with varying frequency or a Schrodinger equation with potential $\frac{R^{\prime \prime}}{R}+\frac{d \omega / d \phi}{2 \omega+3} \frac{\phi^{\prime 2}}{\phi}$

for a particle with energy $k^{2}$ and with the variable $\eta$ playing the role of a spatial co-ordinate. We solve eqn(6) for different epochs of Universe, with equation of state $P=\gamma \rho,-1 \leq \gamma \leq 1$.

The scale factor and the scalar field evolve as power laws of the cosmic time

$$
\begin{gathered}
a(t)=a_{0}\left(\frac{t}{t_{0}}\right)^{\alpha} \\
\phi(t)=\phi_{0}\left(\frac{t}{t_{0}}\right)^{\beta} \\
\omega(t)=\omega(\phi) \approx \phi^{n}=\omega_{0}\left(\frac{t}{t_{0}}\right)^{n \beta}
\end{gathered}
$$

For $\alpha \neq 1$, the translation of eqn(7) and (8) into the conformal time yields $a(\eta)=C_{0}\left(\eta-\overline{\eta_{0}}\right)^{q}$

$\phi(\eta)=\sigma_{0}\left(\eta-\overline{\eta_{0}}\right)^{r}$

$R(\eta)=c_{0}\left(\eta-\overline{\eta_{0}}\right)^{s}$

where $q=\frac{\alpha}{1-\alpha}, r=\frac{\beta}{1-\alpha}, s=q+\frac{r}{2}=\frac{\alpha}{2(1-\alpha)}$ and

$\overline{\eta_{0}} \equiv \eta_{0}-\frac{q}{a_{0} H_{0}}, \eta_{0} \equiv \eta\left(t_{0}\right), H_{0} \equiv H\left(t_{0}\right), c_{0} \equiv a_{0}\left(\frac{a_{0} H_{0}}{q}\right)^{q}, \sigma_{0} \equiv \phi_{0}\left(\frac{a_{0} H_{0}}{q}\right)^{r}$,

$C_{0} \equiv c_{0} \sigma_{0}^{1 / 2}=R_{0}\left(\frac{a_{0} H_{0}}{q}\right)^{s}, R_{0} \equiv a_{0} \phi_{0}^{1 / 2}$.

The wave equation (6)takes the form

$$
\mu^{\prime \prime}(k, \eta)+\left[k^{2}-\frac{s(s-1)}{\left(\eta-\eta_{0}\right)^{2}}-\frac{d \omega / d \phi}{2 \omega+3} \frac{\phi^{\prime 2}}{\phi}\right] \mu(k, \eta)
$$

We take $2 \omega+3=\left(2 \omega_{0}+3\right) \phi^{n}$

This leads to $2 \frac{d \omega}{d \phi}=\left(2 \omega_{0}+3\right) n \phi^{n-1}$. 
This reduces equation (9)to

$$
\mu^{\prime \prime}+\left[k^{2}-\frac{1}{\left(\eta-\overline{\eta_{0}}\right)^{2}}\left(s(s-1)-\frac{n r^{2}}{2}\right)\right] \mu=0
$$

Let $s(s-1)-\frac{n r^{2}}{2}=s^{\prime}\left(s^{\prime}-1\right)$

Where $s^{\prime}=\frac{1}{2} \pm \frac{1}{2} \sqrt{4 s^{2}-4 s+1-2 n r^{2}}$

Hence,we get

$$
\mu^{\prime \prime}+\left[k^{2}-\frac{s^{\prime}\left(s^{\prime}-1\right)}{\left(\eta-\overline{\eta_{0}}\right)^{2}}\right] \mu=0
$$

For very large wave numbers or,more precisely, for waves such that

$$
k^{2} \gg V(\eta), \text { where } V(\eta) \equiv\left|R^{\prime \prime} / R+\frac{d \omega / d \phi}{2 \omega+3} \frac{\phi^{\prime 2}}{\phi}\right| \equiv\left|\frac{s^{\prime}\left(s^{\prime}-1\right)}{\left(\eta-\overline{\eta_{0}}\right)^{2}}\right|,
$$

eqn(10) will have purely oscillatory solutions, and eqn(5) takes the form

$$
h_{i j}^{(k)}(\eta, x)=\frac{1}{a(\eta) \phi^{1 / 2}(\eta)}\left[C_{1} \epsilon^{i k\left(\eta-\overline{\eta_{0}}\right)}+C_{2} \epsilon^{-i k\left(\eta-\overline{\eta_{0}}\right)}\right] \zeta_{i j}(k, x)
$$

where $C_{1}$ and $C_{2}$ are arbitrary constants and the third constant $\overline{\eta_{0}}$ is same as before.We see that the effect of the scalar field $\phi$ is for the scale of amplitude decrease to be set by the function $R(\eta)$ instead of by $a(\eta)$.

In the opposite regime of very small wave numbers, where $k^{2} \ll V(\eta)$,

we obtain,

$$
h_{i j}^{(k)}(\eta, x)=\left[C_{1}+C_{2} \int \eta^{-2}\left(\eta^{\prime}\right) d \eta^{\prime}\right] \zeta_{i j}(k, x)
$$

Note ,however, that ,for bell-shaped potentials, the amplification coefficient will scale as $R\left(\eta_{f}\right) / R\left(\eta_{i}\right)$ instead of $a\left(\eta_{f}\right) / a\left(\eta_{i}\right)$.

The general solution of eqn(10) can be written as[10]

$$
\mu(k, \eta)=k^{-\frac{1}{2}} X^{\frac{1}{2}}\left[C_{1} J_{m}(X)+C_{2} Y_{m}(X)\right]
$$

Here $J_{m}$ and $Y_{m}$ are Bessel and Neumann's functions respectively , $X(\eta)=k\left(\eta-\overline{\eta_{0}}\right)$ and

$m=s^{\prime}-1 / 2$

$= \pm \frac{1}{2} \sqrt{4 s^{2}-4 s+1-2 n r^{2}}$

$= \pm \frac{1}{2} \sqrt{4\left(\frac{2 \alpha+\beta}{2(1-\alpha)}\right)^{2}-4\left(\frac{2 \alpha+\beta}{2(1-\alpha)}\right)+1-2 n\left(\frac{\beta}{1-\alpha}\right)^{2}}$

So,

$$
\begin{gathered}
\mu=\left(\eta-\overline{\eta_{0}}\right)^{1 / 2}\left[C_{1} \sum_{r=0}^{\infty} A_{r}\left(\frac{X}{2}\right)^{m+2 r}+C_{2} \sum_{r=0}^{\infty} B_{r}\left(\frac{X}{2}\right)^{-m+2 r}\right] \\
=\left(\eta-\overline{\eta_{0}}\right)^{1 / 2}\left[C_{1} \sum_{r=0}^{\infty} A_{r}\left(\frac{\pi a(\eta)}{\lambda}\left(\eta-\overline{\eta_{0}}\right)\right)^{m+2 r}+C_{2} \sum_{r=0}^{\infty} B_{r}\left(\frac{\pi a(\eta)}{\lambda}\left(\eta-\overline{\eta_{0}}\right)\right)^{-m+2 r}\right]
\end{gathered}
$$

We see that in order to represent $\mu$ a physical mode the power $m$ has to be real and greater than zero. The difference between the solution (11) and the corresponding one in GR lies in the presence of the coefficient $\beta$ in the parameters $s^{\prime}$ and m.Setting $\beta=0$ we recover the GR solution as expected, since this corresponds to the requirement that $\phi$, and hence $\mathrm{G}$, remains constant. The general cosmological solutions in the framework of GBD theory when matter satisfies the equation of state 
$P=\gamma \rho$ with $-1 \leq \gamma \leq 1$ a constant,were derived by Sahoo and Singh [11].An important feature of these solutions is that they are expressed in terms of the present values of the coupling parameter $\omega$,for vacuum,radiation and matter dominated epochs of the Universe. We write gravitational wave perturbations in different epochs of the Universe by cosidering these solutions in terms of time $t$.

\section{$3.1 \quad$ Vacuum epoch}

For this epoch $\alpha, \beta, n$ are [11]

$$
\begin{aligned}
\alpha & =\frac{1}{\omega_{0}+2}, \beta=-\frac{1}{\omega_{0}+2}, n=-\left(2 \omega_{0}+5\right) \\
\text { As, } d \eta & =\frac{d t}{a}, \text { we find }, \eta \approx\left(\frac{2+\omega_{0}}{1+\omega_{0}}\right) t^{\frac{1+\omega_{0}}{2+\omega_{0}}}
\end{aligned}
$$

And we take, $\left(\eta-\overline{\eta_{0}}\right) \approx\left(\frac{2+\omega_{0}}{1+\omega_{0}}\right) t^{\frac{1+\omega_{0}}{2+\omega_{0}}}$.

Hence,

$$
\begin{gathered}
\mu=\left[C_{1} \sum_{r=0}^{\infty} A_{r}\left(\frac{\pi}{\lambda}\right)^{m+2 r}\left(\frac{2+\omega_{0}}{1+\omega_{0}}\right)^{\frac{1}{2}+(q+1)(m+2 r)} t^{\left(\frac{1+\omega_{0}}{2+\omega_{0}}\right)\left[\frac{1}{2}+(q+1)(m+2 r)\right]}\right]+ \\
{\left[C_{2} \sum_{r=0}^{\infty} B_{r}\left(\frac{\pi}{\lambda}\right)^{-m+2 r}\left(\frac{2+\omega_{0}}{1+\omega_{0}}\right)^{\frac{1}{2}+(q+1)(-m+2 r)} t^{\left(\frac{1+\omega_{0}}{2+\omega_{0}}\right)\left[\frac{1}{2}+(q+1)(-m+2 r)\right]}\right]}
\end{gathered}
$$

It is clear from the above expression that, $\omega_{0} \neq-2$ which is in agreement with the conclusions obtained in our earlier investigation[11].In order to represent $\mu$ a growing modes, the power of $t$ should be real and greater than zero.This gives following conditions

(i) $\omega_{0}>-1$

(ii) $(q+1)(m+2 r)>-\frac{1}{2}$

(iii) $\mathrm{m}$ should be real.

In this case,the order of the Bessel function is $m= \pm \frac{1}{2} \sqrt{1+\frac{2 \omega_{0}+9}{\left(\omega_{0}+1\right)^{2}}}$

As $m$ should be real,we get that $\omega_{0}>-\frac{9}{2}$ which is automatically satisfied if the condition (i) i.e, $\omega_{0}>-1$ is valid.Thus, we conclude that for gravitational waves to grow $\omega_{0}$ should take values greater than -1 .

\subsection{Radiation epoch}

For this epoch $\alpha, \beta$ and $n$ are [11]

$\alpha=\frac{3}{\omega_{0}+6}, \beta=-\frac{3}{\omega_{0}+6}, n=-\left(\frac{2 \omega_{0}+3}{3}\right)$.

As, $d \eta=\frac{d t}{a}$, we find, $\eta \approx\left(\frac{6+\omega_{0}}{3+\omega_{0}}\right) t^{\left(\frac{3+\omega_{0}}{6+\omega_{0}}\right)}$

And we take, $\left(\eta-\overline{\eta_{0}}\right) \approx\left(\frac{6+\omega_{0}}{3+\omega_{0}}\right) t^{\left(\frac{3+\omega_{0}}{6+\omega_{0}}\right)}$.

Hence,

$$
\begin{gathered}
\mu(k, t)=\left[C_{1} \sum_{r=0}^{\infty} A_{r}\left(\frac{\pi}{\lambda}\right)^{m+2 r}\left(\frac{6+\omega_{0}}{3+\omega_{0}}\right)^{\frac{1}{2}+(q+1)(m+2 r)} t^{\left(\frac{3+\omega_{0}}{6+\omega_{0}}\right)\left[\frac{1}{2}+(q+1)(m+2 r)\right]}\right]+ \\
{\left[C_{2} \sum_{r=0}^{\infty} B_{r}\left(\frac{\pi}{\lambda}\right)^{-m+2 r}\left(\frac{6+\omega_{0}}{3+\omega_{0}}\right)^{\frac{1}{2}+(q+1)(-m+2 r)} t^{\frac{3+\omega_{0}}{6+\omega_{0}}\left[\frac{1}{2}+(q+1)(-m+2 r)\right]}\right]}
\end{gathered}
$$

Thus,for gravitational waves to grow during the radiation epoch the above solution should satisfy 
the following conditions,

(i) $\omega_{0}>-3$

(ii) $(q+1)(m+2 r)>-\frac{1}{2}$

(iii)m should be real.

Using the values of $\alpha, \beta$ and $n$ in the expression for $\mathrm{m}$, we get $m= \pm \frac{1}{2} \sqrt{1+\frac{6 \omega_{0}+9}{\left(\omega_{0}+3\right)^{2}}}$. As $\mathrm{m}$ should be real we get $\omega_{0}>-\frac{3}{2}$ which automatically satisfies constraint(i). Thus, for gravitational waves to grow $\omega_{0}$ should take values greater than $-\frac{3}{2}$.

$h_{i j}^{k}=\frac{1}{R} \quad \mu(k, t) \quad \zeta_{i j}(k, t)$

$h_{i j}=\int d^{3} k \quad h_{i j}^{k}(k, t)$

This represents the perturbation in Gravitational field during the radiation epoch.

\subsection{Matter epoch}

During this epoch $\alpha, \beta$, and $n$ are [11]

$\alpha=\frac{2}{\omega_{0}+4}, \beta=-\frac{2}{\omega_{0}+4}, n=-\left(2+\omega_{0}\right)$

As, $d \eta=\frac{d t}{a}$, we find,$\eta \approx\left(\frac{4+\omega_{0}}{2+\omega_{0}}\right) t^{\left(\frac{2+\omega_{0}}{4+\omega_{0}}\right)}$

And we take, $\left(\eta-\overline{\eta_{0}}\right) \approx\left(\frac{4+\omega_{0}}{2+\omega_{0}}\right) t^{\left(\frac{2+\omega_{0}}{4+\omega_{0}}\right)}$.

Hence,

$$
\begin{gathered}
\mu(k, t)=\left[C_{1} \sum_{r=0}^{\infty} A_{r}\left(\frac{\pi}{\lambda}\right)^{m+2 r}\left(\frac{4+\omega_{0}}{2+\omega_{0}}\right)^{\frac{1}{2}+(q+1)(m+2 r)} t^{\left(\frac{2+\omega_{0}}{4+\omega_{0}}\right)\left[\frac{1}{2}+(q+1)(m+2 r)\right]}\right]+ \\
{\left[C_{2} \sum_{r=0}^{\infty} B_{r}\left(\frac{\pi}{\lambda}\right)^{-m+2 r}\left(\frac{4+\omega_{0}}{2+\omega_{0}}\right)^{\frac{1}{2}+(q+1)(-m+2 r)} t^{\left(\frac{2+\omega_{0}}{4+\omega_{0}}\right)\left[\frac{1}{2}+(q+1)(-m+2 r)\right]}\right]}
\end{gathered}
$$

It is clear from the above expression that, $\omega_{0} \neq-2$.Again this result agrees with our earlier conclusions[11].For growing modes, the power of $t$ should be real and greater than zero. This gives following conditions

(i) $\omega_{0}>-2$,

(ii) $(q+1)(m+2 r)>-\frac{1}{2}$

(iii) $m$ should be real.

In this case,the order of the Bessel function, $m$,is $m= \pm \frac{1}{2} \sqrt{1+\frac{12+4 \omega_{0}}{\left(2+\omega_{0}\right)^{2}}}$.

As $m$ should be real,we get $\omega_{0}>-3$.

Thus,for perturbation to grow $\omega_{0}$ should take values greater than -2 . Finally,we get $h_{i j}^{k}=\frac{1}{R} \mu(k, t) \zeta_{i j}(k, t)$ and

$h_{i j}=\int d^{3} k h_{i j}^{k}(k, t)$

This represents the perturbation in gravitational field during the matter epochs.

\section{CONCLUSIONS}

We have solved the gravitational wave equation for a general class of scalar tensor theories of gravity. The solutions are expressed in terms of the present value of the Brans-Dicke coupling parameter i.e, $\omega_{0}$. The solutions represent growing modes when the parameter $\omega_{0}$ takes negative values particularly greater than -1.This negative values of the parameter are required for structure formation,cosmic accleration,superluminal expansion and at the same time a significant amplification 
of perturbations[7, 12].It is also seen that,unlike in GR, there can be amplification of gravitational waves in a radiation -dominated universe. These new features are consequence of the more general scalar tensor theories of gravity which in special cases reduces to Brans-Dicke thory of gravity and GR. In a subsequent paper we will use the results derived here to obtain the spectrum of the relic gravitons in this model.This will enble us to constrain some of the parameters appearing in the scalar -tensor theories. In particular,it could impose a further restriction on the value of the coupling parameter at early times.A small $\omega(\phi)$ is required to have satisfactory nucleation process in some inflationary cosmologies.

\section{Acknowldgment}

The authors are grateful to DST,Govt. of India for providing financial support. The authors also thank Institute of Physics,Bhubaneswar,India for providing facility of the computer center.

\section{References}

[1] F.A.E.Pirani, in Gravitation : An Introduction to Current Research,edited by L.Witten (Wiley, New York 1962); S.W.Hawking,Astrophys.J. 145, 544 (1966);R.A.Issacson, Phys.Rev. 166, 1263 (1968);R.A.Issacson,Phys.Rev. 166, 1272 (1968).

[2] L.P.Grishchuk,Zh.Eksp.Teor.Fiz.67, 825 (1975) [Sov.Phys.JETP 40,409 (1975)];L.P.Grishchuk, Lett.Nuovo Cimento 12, 60 (1975);L.P.Grishchuk,Ann. ～N.Y.Acad.Sci.302,439 (1977);L.P.Grishchuk,Usp.Fiz.Nauk 121,629 (1977) [Sov.Phys.Usp.20,319 (1977)]; L.P.Grishchuk,Usp.Fiz.Nauk.156, 297 (1988) [Sov.Phys.Usp. 31,940 (1989)].

[3] R.V.Wagoher,Phys.Rev.D 1 3209(1970)

[4] J.D.Barrow,J.P.Mimiso and Marcio R.de Garcia Maia, Phys.Rev.D 48 3930(1993); Marcio R de Garcia Maia,Phys.Rev.D48 647 (1993)

[5] B.K.Sahoo and L.P.Singh, Mod.Phys.Lett.A 172409 (2002).

[6] C.M.Will,Theory and Experiment in Gravitational Physics,3rd ed.(Cambridge University Press,Cambridge,England, 1993);K.Nordtvedt,Astrophys.J,161 1059 (1970)

[7] J.P.Baptista, J.C.Fabris and S.V.B.Gonclaves, Astrophys,Space Sc.,296 315 (1996)

[8] S.Weinberg,Gravitation and Cosmology,Wiley,New York (1972).

[9] L.P.Grishchuk and Y.V.Sidorov, Phys.Rev.D,42 3413 (1990);L.P.Grishchuk and M.Solokhin Phys.Rev.D,43 2506 (1991);B.Allen,Phys.Rev.D 372078 (1988);V.Sahni Phys. Rev.D 42453 (1990).

[10] Handbook on Mathematical Functions,edited by M.Abramowitz and I.A.Stegun (Dover,New York,1972)

[11] B.K.Sahoo and L.P.Singh Mod.Phys.Lett.A 182725 (2003).

[12] O.Bertolami and P.J.Martins,Phys.Rev.D,61 064007 (2000) 